\title{
Quantization of nonlocal fractional field theories via the extension problem
}

\author{
A. M. Frassino ${ }^{1}$ and O. Panella $\odot^{2, *}$ \\ ${ }^{1}$ Departament de Física Quàntica i Astrofísica, Institut de Ciències del Cosmos, Universitat de Barcelona, \\ Martí i Franquès 1, E-08028 Barcelona, Spain \\ ${ }^{2}$ Istituto Nazionale di Fisica Nucleare, Sezione di Perugia, Via A. Pascoli, I-06123 Perugia, Italy
}

(Received 13 August 2019; published 10 December 2019)

\begin{abstract}
We use the extension problem proposed by Caffarelli and Silvestre to study the quantization of a scalar nonlocal quantum field theory built out of the fractional Laplacian. We show that the quantum behavior of such a nonlocal field theory in $d$ dimensions can be described in terms of a local action in $d+1$ dimensions which can be quantized using the canonical operator formalism though giving up local commutativity. In particular, we discuss how to obtain the two-point correlation functions and the vacuum energy density of the nonlocal fractional theory as a brane limit of the bulk correlators. We show explicitly how the quantized extension problem reproduces exactly the same particle content of other approaches based on the spectral representation of the fractional propagator. We also briefly discuss the inverse fractional Laplacian and possible applications of this approach in general relativity and cosmology.
\end{abstract}

DOI: 10.1103/PhysRevD.100.116008

\section{INTRODUCTION}

During the past years, there has been an increasing interest in the study of nonlinear and quasilinear equations that involve fractional powers of the Laplacian (see e.g., [1-4]). In particular, fractional powers of the Laplacian are considered in the case of nonlocal diffusion processes described by nonlinear partial differential equations. In such processes, differently from what happens in the standard case for the heat equation, the interactions between particles could be nonlocal, which requires the use of more general operators than the standard Laplacian. The study of nonlinear partial differential equations is complicated also by the fact that there is not a specific approach to investigate the properties of the solutions (e.g., existence, uniqueness, regularity, asymptotic behavior, velocity of propagation, or feasible numerical methods).

In contrast with the usual Laplacian operator, the fractional Laplacian is a nonlocal operator whose value on a function $f(x)$ depends not only on $f(x)$ but also on the value of the function $f\left(x^{\prime}\right)$ on any other point $x^{\prime} \neq x$.

For $\alpha \in(0,2)$ the fractional Laplacian $(-\Delta)^{\frac{\alpha}{2}}$ of a function $f: \mathbb{R}^{n} \rightarrow \mathbb{R}$ can be defined through the Fourier transform as

\footnotetext{
*Corresponding author. orlando.panella@cern.ch

Published by the American Physical Society under the terms of the Creative Commons Attribution 4.0 International license. Further distribution of this work must maintain attribution to the author(s) and the published article's title, journal citation, and DOI. Funded by SCOAP ${ }^{3}$.
}

$$
(-\Delta)^{\frac{\alpha}{2}} f(x)=\int \frac{d^{n} k}{(2 \pi)^{n}}|k|^{\alpha} \tilde{f}(k) e^{i k x},
$$

which, for sufficiently smooth functions, can be rewritten as a singular integral operator as

$$
(-\Delta)^{\frac{\alpha}{2}} f(x)=C_{n, \alpha} \text { P.V. } \int_{\mathbb{R}^{n}} \frac{f(x)-f\left(x^{\prime}\right)}{\left|x-x^{\prime}\right|^{n+\alpha}} d^{n} x^{\prime}
$$

where the real parameter $C_{n, \alpha}=2^{\alpha} \Gamma\left(\frac{n+\alpha}{2}\right) /\left[\pi^{n / 2}|\Gamma(-\alpha / 2)|\right]$ is a normalization constant and P.V. denotes the Cauchy principal value as shown in Refs. [5,6]. However, other authors $[7,8]$ do not explicitly use the principal value notation but they simply imply it. This definition shows that the fractional Laplacian is a nonlocal operator. However, there are also other equivalent possible definitions of the fractional Laplacian [5] that will turn out to be useful in the following discussion. Interestingly, given a function $f: \mathbb{R}^{n} \rightarrow \mathbb{R}$, the following extension problem

$$
\begin{gathered}
u(x, 0)=f(x) \quad x \in \mathbb{R}^{n} \\
\nabla \cdot\left(y^{1-\alpha} \nabla u\right)=0 \quad x \in \mathbb{R}^{n}, y>0,
\end{gathered}
$$

can be solved to obtain a smooth bounded function $u: \mathbb{R}^{n} \times[0, \infty) \rightarrow \mathbb{R}$, where the nonlocal operator $(-\Delta)^{\frac{\alpha}{2}}$ satisfies [7]

$$
(-\Delta)^{\frac{\alpha}{2}} f(x)=+C \lim _{y \rightarrow 0^{+}} y^{1-\alpha} u_{y}(x, y) .
$$

This is a Dirichlet-to-Neumann operator for an appropriate harmonic extension problem and $C$ is a constant that allows 
us to accurately define the limit on the rhs of (4). The constant $C$ is defined as [8]

$$
C=\frac{2^{\alpha} \Gamma[\alpha / 2]}{\alpha \Gamma[-\alpha / 2]} .
$$

The extension problem described above is the mathematical tool that we are going to use in the following sections in order to develop a description of a nonlocal $d$-dimensional scalar field theory, based on the fractional Laplacian, in terms of a local $(d+1)$-dimensional field theory. In particular, this will allows us to establish how the particle content of the fractional nonlocal theory consists of a continuous distribution of massive scalars.

The notable property presented by Eq. (4) is that the nonlocal operator $(-\Delta)^{\frac{\alpha}{2}}$ acting on $f(x)$ in the domain of $\Delta$ is localized through $u$. It has been shown in [8] that the problem can also be extended to the wider range $\alpha \in[0, n]$ (and in [6] that the same extension problem is also valid for negative values of the constant $\alpha$ ). Furthermore, the characterization given in Eq. (4) has also been used to show that the fractional Laplacian $(-\Delta)^{\frac{\alpha}{2}}$ coincides with a certain conformally covariant operator $P_{\frac{\alpha}{2}}$, on the hyperbolic space $\mathbb{R}_{+}^{n+1}$ from which $\mathbb{R}^{n}$ is to be seen as its boundary [8].

In general, the fractional Laplacian has been used in different fields in physics, for example, in solid mechanics to model the elastic behavior of nonlocal continua $[9,10]$, in quantum mechanics and optics [11], or in the context of holography, in models with nonlocality due to charge screening [12-15]. Here, we will also discuss connections to the field of cosmology, and, in particular, to some modified theories of gravity introduced in the past years that either make use of extra dimensions or of the presence of nonlocal terms in the Lagrangian so to model the current phase of cosmological acceleration without resorting to a cosmological constant [16-21]. Interestingly, an analysis of the connection between nonlocal field theories in $d$ dimensions and local field theories in $(d+1)$ dimensions has been explored from the Hamiltonian point of view in [22]. Finally, we would like to mention a nonlocal scalartensor model of gravity [23-26] inspired by the effective action of the $p$-adic string and string field theory on flat spacetime. This model, while addressing a different form of nonlocal operator (exponential of the d'Alambertian $e^{r_{*} \square}$ ), bears similarities with our approach based on the extension problem of [7]. Indeed in [23-26] it has been shown that a wide class of nonlocal theories, including bosonic cubic string field theory, can be identified with local systems living in a higher-dimensional space. This is realized by promoting the parameter $r_{*}$ to a dynamical variable $\left(r_{*} \rightarrow r\right)$ and imposing an appropriate diffusion equation on the new field $\tilde{\phi}(r, x)$ living in $d+1$ dimensions.

In this work, by using the extension problem, it will be shown that quantizing a local field theory in a bounded spacetime (bulk) with an extra spacelike transverse dimension $(y \in[0,+\infty])$ via the operator formalism, but waiving local commutativity, reproduces, through a limiting procedure $(y \rightarrow 0)$, the correlation functions of the nonlocal theory showing a complete equivalence with the fractional propagator obtained independently via analyticity considerations in terms of the spectral representation.

The structure of the paper is the following: In Sec. II we analyze the connection between the bulk local action and the nonlocal action on the brane. In Sec. III we present the quantization of the local action in $(4+1)$ dimensions via the operator formalism. We will see that the two-point bulk correlation functions induce, on the brane, the expected nonlocal correlations functions. In Sec. IV we apply our results to the discussion of the vacuum energy density. As mentioned before, the extension problem has also been shown to be useful in the case of nonlocal terms in the form of the inverse Laplacian. We will study this point in Sec. V. A discussion about other fields is reported in Sec. VI. Finally in Sec. VII we present our conclusions.

\section{NONLOCAL ACTION PROPERTIES}

Although we have in mind physical applications of the Caffarelli-Silvestre extension problem to a fractional scalar field theory in Minkowski spacetime, we stick for the time being to the mathematicians notation and use the $\Delta$ operator. Ordinary results applicable to Minkowski spacetime can be recovered after a Wick rotation of the $x_{0}$ component.

Let us, therefore, consider a nonlocal scalar theory in $d$ Euclidean dimensions. Using the extension problem (3b), the following equality holds:

$$
\begin{aligned}
S & =-\frac{1}{2 C} \int d^{d} x \phi(x)(-\Delta)^{\frac{\alpha}{2}} \phi(x) \\
& =\frac{1}{2} \int d^{d} x \int_{0}^{\infty} d y y^{1-\alpha} \partial_{\mu} \Phi(x, y) \partial^{\mu} \Phi(x, y)
\end{aligned}
$$

where $C$ is the constant (5), $\Phi(x, y)$ denotes the scalar field in $(d+1)$ dimensions, and $\partial_{\mu}$ denotes the derivatives with respect to the $d+1$ variables $(\{x\}, y)$. To prove the equivalence (6) let us start with the following action in $(d+1)$ Euclidean dimensions:

$$
S=\frac{1}{2} \int_{-\infty}^{+\infty} d x \int_{y>0} d y y^{1-\alpha}\left[\partial_{x} \Phi \partial_{x} \Phi+\partial_{y} \Phi \partial_{y} \Phi\right]
$$

then, integration by parts gives 


$$
\begin{aligned}
S= & \frac{1}{2} \int_{0}^{\infty} d y y^{1-\alpha}\left[\left.\Phi \partial_{x} \Phi\right|_{-\infty} ^{+\infty}-\int_{-\infty}^{\infty} d x \Phi \Delta_{x} \Phi\right] \\
& +\frac{1}{2} \int_{-\infty}^{\infty} d x \Phi\left[\left.y^{1-\alpha} \partial_{y} \Phi\right|_{0} ^{+\infty}\right. \\
& \left.-\int_{0}^{+\infty} d y\left[(1-\alpha) y^{-\alpha} \partial_{y} \Phi+y^{1-\alpha} \Delta_{y} \Phi\right]\right] .
\end{aligned}
$$

Now, supposing that the field $\Phi(x, y)$ at $x^{i}= \pm \infty$ and $y=+\infty$ is zero, we are left only with

$$
\begin{aligned}
S= & -\frac{1}{2} \int_{-\infty}^{\infty} d x \int_{0}^{+\infty} d y \Phi \\
& \times\left[\Delta_{x} \Phi+(1-\alpha) y^{-\alpha} \partial_{y} \Phi+y^{1-\alpha} \Delta_{y} \Phi\right]+ \\
& -\frac{1}{2} \int_{-\infty}^{\infty} d x \lim _{y \rightarrow 0^{+}}\left[y^{1-\alpha} \Phi \partial_{y} \Phi\right]
\end{aligned}
$$

and using Eq. (3a) and Eq. (3b)

$$
\operatorname{div}\left(y^{1-\alpha} \nabla u\right)=y^{1-\alpha} u_{x x}+y^{1-\alpha} u_{y y}+(1-\alpha) y^{-\alpha} u_{y}=0
$$

in the first line of Eq. (9) and then, substituting Eq. (4) in the second line, the action (9) reduces to

$$
S=-\frac{1}{2 C} \int_{-\infty}^{\infty} d x \phi(x)(-\Delta)^{\frac{\alpha}{2}} \phi(x)
$$

Hence, using the extension problem, a nonlocal theory can be described in terms of a local action in a $(d+1)$ spacetime as expressed in Eq. (6). The equivalence (6) relates, therefore, a local quantum field theory in the bulk (hyperbolic) space $\mathbb{R}_{+}^{n+1}$ to a nonlocal quantum field theory on the nontrivial boundary $\mathbb{R}^{n}$ [27].

It should be noted indeed that the coefficient present in the $(d+1)$-dimensional part of Eq. (6) can be associated to the half space $(y>0)$ representation of an anti-de Sitter spacetime (or hyperbolic space if Wick rotated):

$$
\begin{aligned}
d s^{2} & =\frac{1}{y^{2}}\left(-d t^{2}+d y^{2}+\sum_{i=2}^{d} d x_{i}^{2}\right) \\
& =\left(\frac{1}{y^{2}} \eta_{\mu \nu}\right) d x^{\mu} d x^{\nu}=g_{\mu \nu} d x^{\mu} d x^{\nu}
\end{aligned}
$$

Therefore, starting from the rhs of Eq. (6) follows

$$
\begin{aligned}
y^{1-\alpha} \eta^{\mu \nu} \partial_{\mu} \Phi \partial_{\nu} \Phi & =y^{1-\alpha-2} y^{2} \eta^{\mu \nu} \partial_{\mu} \Phi \partial_{\nu} \Phi \\
& =\sqrt{\operatorname{det} g} g^{\mu \nu} \partial_{\mu} \Phi \partial_{\nu} \Phi
\end{aligned}
$$

where the last equality is valid only if $\alpha=d$.

\section{A. Variation of the nonlocal action}

Here let us consider the nonlocal action by itself and derive the corresponding equation of motion (EOM). by computing the variation of the action:

$$
S=\frac{1}{2 C} \int d^{4} x \phi(x)(-\Delta)^{\frac{\alpha}{2}} \phi(x)
$$

under an arbitrary variation of the field $\delta \phi(x)$ :

$$
\phi(x) \rightarrow \phi(x)+\delta \phi(x) .
$$

The variation of the action to linear order in $\delta \phi(x)$ is given by

$$
\begin{aligned}
\delta S= & S[\phi+\delta \phi]-S[\phi] \\
= & \frac{1}{2 C} \int d^{4} x(\phi(x)+\delta \phi(x))(-\Delta)^{\frac{\alpha}{2}}(\phi(x)+\delta \phi(x)) \\
& -\frac{1}{2 C} \int d^{4} x \phi(x)(-\Delta)^{\frac{\alpha}{2}} \phi(x) \\
= & \frac{1}{2 C} \int d^{4} x \phi(x)(-\Delta)^{\frac{\alpha}{2}} \delta \phi(x) \\
& +\frac{1}{2 C} \int d^{4} x \delta \phi(x)(-\Delta)^{\frac{\alpha}{2}} \phi(x)+\mathcal{O}\left(\delta \phi^{2}\right) .
\end{aligned}
$$

Now we use the distributional definition of the fractional Laplacian stated in (b) of Theorem 1.1 of [5]:

$$
\int d^{4} x f(x)(-\Delta)^{\frac{\alpha}{2}} g(x)=\int d^{4} x g(x)(-\Delta)^{\frac{\alpha}{2}} f(x),
$$

in order to show that the first term on the rhs of Eq. (16) is the same as the second [with the Laplacian acting on the field $\phi(x)$ ]. So Eq. (16) becomes

$$
\delta S=\frac{1}{C} \int d^{4} x \delta \phi(x)(-\Delta)^{\frac{\alpha}{2}} \phi(x)+\mathcal{O}\left(\delta \phi^{2}\right)
$$

and the EOM are obtained requiring that the first order (in $\delta \phi$ ) variation of the action vanishes. Since the variation is arbitrary we obtain the EOM as

$$
(-\Delta)^{\frac{\alpha}{2}} \phi(x)=0,
$$

i.e., the fractional Laplace equation (19) sets an important condition: it corresponds to the particular case in which the rhs of Eq. (4) is equal to zero. The EOM, therefore, sets the following boundary condition for the quantum field $\Phi(x, y)$ propagating in the bulk space with the extra dimension $y$ :

$$
\lim _{y \downarrow 0} y^{1-\alpha} \Phi_{y}(x, y)=0 .
$$

This boundary condition will play a crucial role in the quantization of the theory. Some aspects of boundary 
conditions in the bulk and their effect on the field dynamics on the brane have been analyzed in $[28,29]$. In particular in [29] it is shown how a large family of bulk fields quantized giving up local commutativity induces brane fields (in the limit $y \rightarrow 0$ ) which do verify it.

\section{QUANTIZATION OF THE EXTENDED PROBLEM IN $(d+1)$ DIMENSIONS}

In this section we perform a quantization of the local action in $(4+1)$ dimensions via the operator formalism and show that its two-point bulk correlation functions induce, on the brane, the expected nonlocal correlation functions as in Eq. (63).

\section{A. Eigenfunctions}

We now switch to Minkowski spacetime performing a Wick rotation $x_{0}=i c t$ in Eq. (6) and obtain

$$
\begin{aligned}
S & =\frac{1}{2 C} \int d^{d} x \phi(x) \square^{\frac{\alpha}{2}} \phi(x) \\
& =\frac{1}{2} \int d^{d} x \int_{0}^{\infty} d y y^{1-\alpha} \partial_{\mu} \Phi(x, y) \partial^{\mu} \Phi(x, y) .
\end{aligned}
$$

Let us therefore start with the following action for a massless scalar field $\Phi(x, y)$ in the bulk:

$$
S=\frac{1}{2} \int d^{d} x \int_{0}^{\infty} d y y^{1-\alpha} \partial_{\mu} \Phi(x, y) \partial^{\mu} \Phi(x, y),
$$

the variation of the action gives the equation of motion $\partial^{\mu}\left(y^{1-\alpha} \partial_{\mu} \Phi(x, y)\right)=0$, which explicitly reads

$\square_{x} \Phi(x, y)-(1-\alpha) \frac{1}{y} \partial_{y} \Phi(x, y)-\partial_{y}^{2} \Phi(x, y)=0$,

where $\square_{x}=\partial_{0}^{2}-\nabla^{2}$. Equation (23) can be solved by the separation of variables, writing $\Phi(x, y)=\varphi(x) \psi(y)$, from which follows

$$
\begin{aligned}
\frac{1}{\varphi(x)} \square_{x} \varphi(x) & =\frac{1}{\psi(y)}\left[\partial_{y}^{2}+(1-\alpha) \frac{1}{y} \partial_{y}\right] \psi(y) \\
& =\text { const }=-\lambda^{2},
\end{aligned}
$$

where $\lambda$ has dimension of mass. Therefore, from the EOM, one gets the following equations:

$$
\begin{gathered}
\left(\square_{x}+\lambda^{2}\right) \varphi(x)=0, \\
{\left[\partial_{y}^{2}+(1-\alpha) \frac{1}{y} \partial_{y}\right] \psi(y)=-\lambda^{2} \psi(y) .}
\end{gathered}
$$

The first one, Eq. (25a), gives the usual expansion of the field $\varphi(x)$ in plane waves of mass $\lambda$, whereas the second, Eq. (25b), can be solved imposing the appropriate boundary condition:

$$
\lim _{y \rightarrow 0} y^{1-\alpha} \partial_{y} \psi(y)=0
$$

This boundary condition on the $y$-dependent part of the wave function comes from the condition in Eq. (4) on the field $\Phi(x, y)$. The equation for the wave function $\psi(y)$ can be reduced to a Schrödinger problem with a potential of the type $1 / y^{2}$ (see [30]). Therefore, in order to find the eigenfunctions $\psi(y)$, one can proceed in the following way. The first step is to change the variable $t=\lambda y$ in Eq. (25b)

$$
\left[\lambda^{2} \partial_{t}^{2}+(1-\alpha) \frac{\lambda}{t} \lambda \partial_{t}\right] \psi=-\lambda^{2} \psi
$$

Then, writing $\psi(t)=t^{\frac{\alpha}{2}} \chi(t)$, one gets the Bessel equation

$$
t^{2} \partial_{t}^{2} \chi(t)+t \partial_{t} \chi(t)+\left[t^{2}-\left(\frac{\alpha}{2}\right)^{2}\right] \chi(t)=0,
$$

whose general solution is the linear combination of $J_{\alpha / 2}(t)$ and $Y_{\alpha / 2}(t)$ :

$$
\psi(\lambda, y)=t^{\frac{\alpha}{2}}\left[A J_{\alpha / 2}(t)+B Y_{\alpha / 2}(t)\right]_{t=\lambda y} .
$$

The two constants $A$ and $B$ in Eq. (29) can be fixed imposing the boundary conditions

$$
\lim _{y \rightarrow 0} \psi(\lambda, y)=1, \quad \lim _{y \rightarrow 0} y^{1-\alpha} \partial_{y} \psi(\lambda, y)=0 .
$$

Using the asymptotic expansion for $t \rightarrow 0$ (i.e., $y \rightarrow 0$ )

$$
\begin{gathered}
J_{\alpha / 2}(t) \simeq \frac{t^{\frac{\alpha}{2}}}{2^{2} \Gamma\left(\frac{\alpha}{2}+1\right)}, \\
Y_{\alpha / 2}(t) \simeq-\frac{2^{\frac{\alpha}{2}} \Gamma\left(\frac{\alpha}{2}\right) t^{-\frac{\alpha}{2}}}{\pi}+\frac{\cot \left(\frac{\alpha}{2} \pi\right) t^{\frac{\alpha}{2}}}{\Gamma\left(\frac{\alpha}{2}+1\right) 2^{\frac{\alpha}{2}}},
\end{gathered}
$$

the solution (29) reads

$$
\psi(\lambda, y) \simeq \frac{\left[A+B \cot \left(\pi \frac{\alpha}{2}\right)\right] t^{\alpha}}{\Gamma\left(\frac{\alpha}{2}+1\right) 2^{\frac{\alpha}{2}}}-\frac{B \Gamma\left(\frac{\alpha}{2}\right) 2^{\frac{\alpha}{2}}}{\pi} .
$$

Then, the first boundary condition in (30) gives

$$
\lim _{y \rightarrow 0} \psi(\lambda, y)=-\frac{B \Gamma\left(\frac{\alpha}{2}\right) 2^{\frac{\alpha}{2}}}{\pi}=1,
$$


and the second boundary condition in Eq. (30) gives

$$
\lim _{y \rightarrow 0} y^{1-\alpha} \partial_{y} \psi(\lambda, y)=0 \Leftrightarrow A+B \cot \left(\pi \frac{\alpha}{2}\right)=0 .
$$

Thus, the eigenfunctions (29) read

$$
\psi(\lambda, y)=\frac{\pi(\lambda y)^{\frac{\alpha}{2}}}{\Gamma\left(\frac{\alpha}{2}\right) 2^{\frac{\alpha}{2}}}\left[\cot \left(\pi \frac{\alpha}{2}\right) J_{\alpha / 2}(\lambda y)-Y_{\alpha / 2}(\lambda y)\right] .
$$

Now, using the definition

$$
Y_{\nu}(z)=J_{\nu} \cot (\pi \nu)-\frac{1}{\sin (\pi \nu)} J_{-\nu}(z)
$$

follows the identity

$$
J_{\alpha / 2}(\lambda y) \cot \left(\pi \frac{\alpha}{2}\right)-Y_{\alpha / 2}(\lambda y)=\frac{1}{\sin \left(\pi \frac{\alpha}{2}\right)} J_{-\alpha / 2}(\lambda y)
$$

that, used in (36), gives the final form

$$
\psi(\lambda, y)=\frac{\pi}{2^{\frac{\alpha}{2}} \Gamma\left(\frac{\alpha}{2}\right) \sin \left(\pi \frac{\alpha}{2}\right)}(\lambda y)^{\frac{\alpha}{2}} J_{-\alpha / 2}(\lambda y) .
$$

This result can be compared with the final result of Sec. 3.2 of [6], where the authors study the existence and uniqueness results for the extension problem. Note that the eigenfunctions (39) satisfy the boundary conditions

$$
\psi(\lambda, 0)=1,\left.\quad y^{1-\alpha} \partial_{y} \psi(\lambda, y)\right|_{y=0}=0
$$

as required in (30). These eigenfunctions form an orthonormal system and satisfy the completeness relation

$$
\begin{aligned}
\int & d \lambda \mu(\lambda) \bar{\psi}\left(\lambda, y_{1}\right) \psi\left(\lambda, y_{2}\right) \\
& =\frac{\left(2^{-\frac{\alpha}{2}} \pi\right)^{2} y_{1}^{\frac{\alpha}{2}} y_{2}^{\frac{\alpha}{2}}}{\left[\Gamma\left(\frac{\alpha}{2}\right) \sin \left(\frac{\pi \alpha}{2}\right)\right]^{2}} \int_{0}^{\infty} d \lambda \lambda^{\alpha} \mu(\lambda) J_{-\frac{\alpha}{2}}\left(\lambda y_{1}\right) J_{-\frac{\alpha}{2}}\left(\lambda y_{2}\right) .
\end{aligned}
$$

For the Bessel functions, the following identity [31] holds [32] for $\nu>-1$ :

$$
\int_{0}^{\infty} d \lambda \lambda J_{\nu}\left(\lambda y_{1}\right) J_{\nu}\left(\lambda y_{2}\right)=\frac{1}{y_{1}} \delta\left(y_{1}-y_{2}\right) .
$$

We now consider a function $\mu(\lambda)$ in Eq. (41) such that

$$
\left[\frac{2^{-\frac{\alpha}{2}} \pi}{\Gamma\left(\frac{\alpha}{2}\right) \sin \left(\pi \frac{\alpha}{2}\right)}\right]^{2} \mu(\lambda) \lambda^{\alpha}=\lambda
$$

so that

$$
\mu(\lambda)=\left[\frac{2^{-\frac{\alpha}{2}} \pi}{\Gamma\left(\frac{\alpha}{2}\right) \sin \left(\pi \frac{\alpha}{2}\right)}\right]^{-2} \lambda^{1-\alpha}
$$

Then the eigenfunctions satisfy the completeness relation:

$$
\begin{aligned}
\int_{0}^{\infty} d \lambda \mu(\lambda) \bar{\psi}\left(\lambda, y_{1}\right) \psi\left(\lambda, y_{2}\right) & =\frac{\left(y_{1} y_{2}\right)^{\frac{\alpha}{2}}}{y_{1}} \delta\left(y_{1}-y_{2}\right) \\
& =y_{1}^{\alpha-1} \delta\left(y_{1}-y_{2}\right) .
\end{aligned}
$$

This particular choice of the function $\mu(\lambda)$ is due to our interest in describing conformal (scale) invariance on the brane. As it will be clear in the following section, the function $\mu(\lambda)$ plays a central role in the quantization of the theory. It will ensure the canonical equal-time commutation relation between the bulk field and its conjugate momentum. As discussed in [29], in general, other possibilities could be considered for the function $\mu(\lambda)$ beyond the one in Eq. (44) but requiring scale invariance fixes it to the specific form $\mu(\lambda) \propto \lambda^{1-\alpha}$. This will be discussed again in connection with massive models of gravity in Sec. VI.

\section{B. Quantization rules and field expansion}

The field decomposition over its normal modes is

$$
\begin{aligned}
\Phi(x, y)= & \int \frac{d^{3} \boldsymbol{p}}{(2 \pi)^{3}} \int_{0}^{+\infty} d \lambda \mu(\lambda)\left[a(\boldsymbol{p}, \lambda) \frac{e^{i\left[\boldsymbol{p} \cdot \boldsymbol{x}-\omega_{\lambda^{2}}(\boldsymbol{p}) t\right]}}{\sqrt{2 \omega_{\lambda^{2}}(\boldsymbol{p})}} \psi(y, \lambda)\right. \\
& \left.+a^{\dagger}(\boldsymbol{p}, \lambda) \frac{e^{-i\left[\boldsymbol{p} \cdot \boldsymbol{x}-\omega_{\lambda^{2}}(\boldsymbol{p}) t\right]}}{\sqrt{2 \omega_{\lambda^{2}}(\boldsymbol{p})}} \psi^{*}(y, \lambda)\right]
\end{aligned}
$$

where $\omega_{\lambda^{2}}(\boldsymbol{p})=\sqrt{\boldsymbol{p}^{2}+\lambda^{2}}$, and $a(\boldsymbol{p}, \lambda), a^{\dagger}(\boldsymbol{p}, \lambda)$ are creation and annihilation operators for the modes of momentum $\boldsymbol{p}$ and mass $\lambda$ with quantization rules [29]:

$$
\begin{gathered}
{\left[a(\boldsymbol{p}, \lambda), a^{\dagger}(\boldsymbol{p}, \lambda)\right]=(2 \pi)^{3} \delta^{3}\left(\boldsymbol{p}-\boldsymbol{p}^{\prime}\right) \frac{1}{\mu(\lambda)} \delta\left(\lambda-\lambda^{\prime}\right)} \\
{[a(\boldsymbol{p}, \lambda), a(\boldsymbol{p}, \lambda)]=0} \\
{\left[a^{\dagger}(\boldsymbol{p}, \lambda), a^{\dagger}(\boldsymbol{p}, \lambda)\right]=0 .}
\end{gathered}
$$

To find the equal-time commutation relations, we start from Eq. (46) and take the derivative of the field:

$$
\begin{aligned}
\partial_{0} \Phi(x, y)= & \int \frac{d^{3} \boldsymbol{p}}{(2 \pi)^{3}} \int_{0}^{+\infty} d \lambda \mu(\lambda)\left[a(\boldsymbol{p}, \lambda) \frac{e^{i\left[\boldsymbol{p} \cdot \boldsymbol{x}-\omega_{\lambda^{2}}(\boldsymbol{p}) t\right]}}{\sqrt{2 \omega_{\lambda^{2}}(\boldsymbol{p})}}\right. \\
& \times\left[-i \omega_{\lambda^{2}}(\boldsymbol{p})\right] \psi(y, \lambda)+a^{\dagger}(\boldsymbol{p}, \lambda) \frac{e^{-i\left[\boldsymbol{p} \cdot \boldsymbol{x}-\omega_{\lambda^{2}}(\boldsymbol{p}) t\right]}}{\sqrt{2 \omega_{\lambda^{2}}(\boldsymbol{p})}} \\
& \left.\times\left[i \omega_{\lambda^{2}}(\boldsymbol{p})\right] \psi^{*}(y, \lambda)\right] .
\end{aligned}
$$

The conjugate momentum of $\Phi$, derived from the Lagrangian density of the local action in Eq. (22), is

$$
\Pi(t, \boldsymbol{r}, y)=y^{1-\alpha} \partial_{t} \Phi(t, \boldsymbol{r}, y) .
$$

We then compute 


$$
\begin{aligned}
& {\left[\partial_{0} \Phi(\boldsymbol{r}, y, t), \Phi\left(\boldsymbol{r}^{\prime}, y^{\prime}, t\right)\right]=\frac{1}{2} \int \frac{d^{3} \boldsymbol{p}}{(2 \pi)^{3}} \int \frac{d^{3} \boldsymbol{p}^{\prime}}{(2 \pi)^{3}} \int_{0}^{+\infty} d \lambda \mu(\lambda) \int_{0}^{+\infty} d \lambda^{\prime} \mu\left(\lambda^{\prime}\right)} \\
& \times\left[a(\boldsymbol{p}, \lambda) \psi(y, \lambda) e^{i\left[\boldsymbol{p} \cdot \boldsymbol{r}-\omega_{\lambda^{2}}(\boldsymbol{p}) t\right]}(-i) \sqrt{\omega_{\lambda^{2}}(\boldsymbol{p})}+a^{\dagger}(\boldsymbol{p}, \lambda) \psi^{*}(y, \lambda) e^{-i\left[\boldsymbol{p} \cdot \boldsymbol{r}-\omega_{\lambda^{2}}(\boldsymbol{p}) t\right]} i \sqrt{\omega_{\lambda^{2}}(\boldsymbol{p})},\right. \\
& \left.\times a\left(\boldsymbol{p}^{\prime}, \lambda^{\prime}\right) \psi\left(y^{\prime}, \lambda^{\prime}\right) \frac{e^{i\left[\boldsymbol{p}^{\prime} \cdot \boldsymbol{r}^{\prime}-\omega_{\lambda^{\prime 2}}\left(\boldsymbol{p}^{\prime}\right) t\right]}}{\sqrt{\omega_{\lambda^{\prime 2}}\left(\boldsymbol{p}^{\prime}\right)}}+a^{\dagger}\left(\boldsymbol{p}^{\prime}, \lambda^{\prime}\right) \psi^{*}\left(y^{\prime}, \lambda^{\prime}\right) \frac{e^{-i\left[\boldsymbol{p}^{\prime} \cdot \boldsymbol{r}^{\prime}-\omega_{\lambda^{\prime 2}}\left(\boldsymbol{p}^{\prime}\right) t\right]}}{\sqrt{\omega_{\lambda^{\prime 2}}\left(\boldsymbol{p}^{\prime}\right)}}\right] .
\end{aligned}
$$

Applying the quantization rules (47a)-(47c), only two of the four terms are nonvanishing, those corresponding, respectively, to the commutators $\left[a(\boldsymbol{p}, \lambda), a^{\dagger}\left(\boldsymbol{p}^{\prime}, \lambda^{\prime}\right)\right]$ and $\left[a^{\dagger}(\boldsymbol{p}, \lambda), a\left(\boldsymbol{p}^{\prime}, \lambda^{\prime}\right)\right]$, and one gets

$$
\begin{aligned}
{\left[\partial_{0} \Phi(\boldsymbol{r}, y, t), \Phi\left(\boldsymbol{r}^{\prime}, y^{\prime}, t\right)\right]=} & \frac{(-i)}{2} \int \frac{d^{3} \boldsymbol{p}}{(2 \pi)^{3}} \int \frac{d^{3} \boldsymbol{p}^{\prime}}{(2 \pi)^{3}} \int_{0}^{+\infty} d \lambda \mu(\lambda) \int_{0}^{+\infty} d \lambda^{\prime} \mu\left(\lambda^{\prime}\right) \\
& \times(2 \pi)^{3} \delta^{3}\left(\boldsymbol{p}-\boldsymbol{p}^{\prime}\right) \frac{1}{\mu(\lambda)} \delta\left(\lambda-\lambda^{\prime}\right)\left[\psi(y, \lambda) \psi^{*}\left(y^{\prime}, \lambda^{\prime}\right) e^{i \boldsymbol{p} \cdot\left(\boldsymbol{r}-\boldsymbol{r}^{\prime}\right)} e^{-i \omega_{\lambda}^{2}(\boldsymbol{p}, \lambda) t} e^{+i \omega_{\lambda^{\prime 2}}\left(\boldsymbol{p}^{\prime}, \lambda^{\prime}\right) t}\right. \\
& \left.+\psi^{*}\left(y^{\prime}, \lambda^{\prime}\right) \psi(y, \lambda) e^{-i \boldsymbol{p}^{\prime} \cdot\left(\boldsymbol{r}-\boldsymbol{r}^{\prime}\right)} e^{+i \omega_{\lambda}^{2}(\boldsymbol{p}, \lambda) t} e^{-i \omega_{\lambda^{\prime 2}}\left(\boldsymbol{p}^{\prime}, \lambda^{\prime}\right) t}\right] .
\end{aligned}
$$

Finally, eliminating the integrals $\int d^{3} \boldsymbol{p}^{\prime}$ and $\int d \lambda^{\prime}$, thanks to the Dirac delta functions, one is left with

$$
\left[\partial_{0} \Phi(\boldsymbol{r}, y, t), \Phi\left(\boldsymbol{r}^{\prime}, y^{\prime}, t\right)\right]=\frac{(-i)}{2} \int \frac{d^{3} \boldsymbol{p}}{(2 \pi)^{3}} \int_{0}^{+\infty} d \lambda \mu(\lambda)\left[\psi(y, \lambda) \psi^{*}\left(y^{\prime}, \lambda\right) e^{i \boldsymbol{p} \cdot\left(\boldsymbol{r}-\boldsymbol{r}^{\prime}\right)}+\psi^{*}\left(y^{\prime}, \lambda\right) \psi(y, \lambda) e^{-i \boldsymbol{p} \cdot\left(\boldsymbol{r}-\boldsymbol{r}^{\prime}\right)}\right]
$$

Upon use of the completeness relations (45), this becomes

$$
\left[\partial_{0} \Phi(\boldsymbol{r}, y, t), \Phi\left(\boldsymbol{r}^{\prime}, y^{\prime}, t\right)\right]=\frac{(-i)}{2} \delta^{3}\left(\boldsymbol{r}-\boldsymbol{r}^{\prime}\right)\left[y^{\alpha-1} \delta\left(y-y^{\prime}\right)+\left(y^{\prime}\right)^{\alpha-1} \delta\left(y^{\prime}-y\right)\right]
$$

This can be rewritten as

$$
\left[y^{1-\alpha} \partial_{0} \Phi(\boldsymbol{r}, y, t), \Phi\left(\boldsymbol{r}^{\prime}, y^{\prime}, t\right)\right]=-i \delta^{3}\left(\boldsymbol{r}-\boldsymbol{r}^{\prime}\right) \delta\left(y-y^{\prime}\right)
$$

which is, on account of Eq. (49), the standard canonical equal-time commutation relation between the field's conjugate momentum $\Pi(\boldsymbol{r}, y, t)$ and the field $\Phi(\boldsymbol{r}, y, t)$ itself:

$$
\left[\Pi(\boldsymbol{r}, y, t), \Phi\left(\boldsymbol{r}^{\prime}, y^{\prime}, t\right)\right]=-i \delta^{3}\left(\boldsymbol{r}-\boldsymbol{r}^{\prime}\right) \delta\left(y-y^{\prime}\right) .
$$

This warrants that our $(4+1)$-dimensional theory with one extra space dimension is a standard local quantum field theory with canonical quantization. We emphasize how the possibility of carrying out the canonical quantization rests upon the completeness relation, Eq. (45), satisfied by the eigenfunctions of the equation of motion along the extra dimension with the appropriate function $\mu(\lambda)$. Note that we cannot take the limit of Eqs. (55) and (54) on the brane as we know that in the nonlocal $(3+1)$-dimensional theory, the very concept of the conjugate momentum field is ill defined.

\section{Causal structure}

Let us consider now the commutator, at different spacetime points, of the bulk fields, $\left[\Phi\left(x_{1}, y_{1}\right), \Phi\left(x_{2}, y_{2}\right)\right]$. This quantity can be computed using the field expansion in
Eq. (46) and the quantization rules in Eqs. (47a)-(47c). Then, making use of the identity,

$$
\frac{1}{2 \omega_{\lambda^{2}}(\boldsymbol{p})}=\int d p_{0} \Theta\left(p_{0}\right) \delta\left(\boldsymbol{p}^{2}-\lambda^{2}\right)
$$

we find

$$
\begin{aligned}
& {\left[\Phi\left(x_{1}, y_{1}\right), \Phi\left(x_{2}, y_{2}\right)\right]} \\
& =\int d \lambda \mu(\lambda) \psi\left(y_{1}, \lambda\right) \psi^{*}\left(y_{2}, \lambda\right) \int \frac{d^{3} \boldsymbol{p}}{(2 \pi)^{3}} d p_{0} \theta\left(p_{0}\right) \delta\left(\boldsymbol{p}^{2}-\lambda^{2}\right) \\
& \quad \times\left[e^{+i\left[\boldsymbol{p} \cdot\left(\boldsymbol{x}_{1}-\boldsymbol{x}_{2}\right)-p_{0}\left(t_{1}-t_{2}\right)\right]}-e^{-i\left[\boldsymbol{p} \cdot\left(\boldsymbol{x}_{1}-\boldsymbol{x}_{2}\right)-p_{0}\left(t_{1}-t_{2}\right)\right]}\right]
\end{aligned}
$$

where $x_{1}=\left(t_{1}, \boldsymbol{x}_{1}\right)$ and $x_{2}=\left(t_{2}, \boldsymbol{x}_{2}\right)$. Recalling the definition of the Pauli-Jordan function

$$
D_{\lambda^{2}}(x)=i \int \frac{d^{4} p}{(2 \pi)^{4}} e^{-i p \cdot x} 2 \pi \delta\left(p^{2}-\lambda^{2}\right)\left[\theta\left(p^{0}\right)-\theta\left(-p^{0}\right)\right]
$$

we finally obtain 


$$
\begin{aligned}
& {\left[\Phi\left(x_{1}, y_{1}\right), \Phi\left(x_{2}, y_{2}\right)\right]} \\
& \quad=(-i) \int_{0}^{+\infty} d \lambda \mu(\lambda) \psi^{*}\left(y_{1}, \lambda\right) \psi\left(y_{2}, \lambda\right) D_{\lambda^{2}}\left(x_{1}-x_{2}\right) .
\end{aligned}
$$

Local commutativity implies that the above field commutator for bulk spacelike intervals between the points $\left(x_{1}, y_{1}\right)$ and $\left(x_{2}, y_{2}\right)$ would vanish identically. As also discussed in [29], for general functions $\mu(\lambda)$ this is not the case in Eq. (59). Therefore, in spite of the fact that the bulk field is built out of a classical local action at the quantum level the field is nonlocal (local commutativity is lost). The origin of this very peculiar property is ultimately due to the defect introduced in the bulk by the presence of the brane. The fact that the function $\mu(\lambda)$ appears in the quantization rules in Eq. (47) shows that this is a genuine quantum phenomenon.

Taking the brane limit of Eq. (59) on account of the boundary condition $\psi(y, \lambda) \rightarrow 1$ as $y \rightarrow 0$ we find

$$
\left[\phi\left(x_{1}\right), \phi\left(x_{2}\right)\right]=-i \int_{0}^{+\infty} d \lambda \mu(\lambda) D_{\lambda^{2}}\left(x_{1}-x_{2}\right) .
$$

For spacelike separations, $\left(x_{1}-x_{2}\right)$, the Pauli-Jordan function $D_{\lambda^{2}}\left(x_{1}-x_{2}\right)$ vanishes identically for any $\lambda$ (for it is the Pauli-Jordan function of a scalar field of mass $\lambda$ ) and therefore the field commutator will vanish. Thus, the brane field satisfies local commutativity. On the other end, the brane field is a noncanonical quantum field in the sense that it is associated to a nonlocal classical action and it cannot be quantized canonically. Indeed, we have seen that the brane limit of the (canonical) equal-time commutation relation in the bulk, Eq. (54), is ill defined.

\section{Two-point correlation functions-the Wightman function and the Feynman propagator-from the extension problem}

The nonlocal action in Eq. (14) as well as the EOM in Eq. (19) seems to be directly related to the unparticle propagator introduced in [33] in the context of the study of a massive scale invariant theory. This can be easily seen by considering the Green's function of Eq. (19) defined in the usual way:

$$
(-\Delta)^{\frac{\alpha}{2}} G\left(x, x^{\prime}\right)=\delta^{4}\left(x-x^{\prime}\right)
$$

The above equation is straightforwardly solved in Fourier space using (1),

$$
(-\Delta)^{\frac{\alpha}{2}} G\left(x-x^{\prime}\right)=\int \frac{d^{4} p}{(2 \pi)^{4}}\left(p^{2}\right)^{\frac{\alpha}{2}} G(p) e^{i p \cdot\left(x-x^{\prime}\right)} .
$$

Then from Eq. (61) we obtain

$$
G(p)=\left(p^{2}\right)^{-\alpha / 2}=\left(\sqrt{p^{2}}\right)^{-\alpha}
$$

This is exactly the unparticle propagator discussed, for instance, in Georgi's paper [34] with $\alpha / 2=2-d_{\mathcal{U}}$ (up to an irrelevant numerical constant), where $d_{\mathcal{U}}$ is the scaling dimension of the unparticle operator in the low-energy theory. Indeed, the unparticle propagator is obtained requiring scale invariance in the low-energy effective field theory of a theory with a nontrivial scale invariant IR fixed point [33]. Therefore, the unparticle propagator seems to be a feature of a nonlocal action of the type in Eq. (14).

The particle content of the theory described by the fractional propagator in Eq. (63) can be derived by computing a spectral density representation in terms of the discontinuities across the branch cut. For any analytic function $f(z)=\int_{-\infty}^{+\infty} d t \rho(t) \frac{1}{z-t}$ the discontinuity across the branch cut is as follows: $\operatorname{disc}[f(z)]=\{f(z+i \epsilon)-$ $f(z-i \epsilon)\}_{\epsilon \rightarrow 0}=-2 \pi i \rho(z)$. We define the argument of the complex $z$ variable, $\theta$, to vary in the interval $-\pi<\theta<$ $\pi$ so that the function $\sqrt{z}$ has a branch cut along the negative (real) $z$ axis. We therefore let $z=-p^{2}$ in Eq. (63) and the fractional propagator $(\sqrt{-z})^{-\alpha}$ will have a discontinuity for positive $z$. Explicitly we find

$$
\operatorname{disc}\left[(\sqrt{-z})^{-\alpha}\right]=\left\{\begin{array}{ll}
2 i \sin \left(\frac{\alpha \pi}{2}\right)(\sqrt{z})^{-\alpha} & z>0 \\
0 & z \leq 0
\end{array} .\right.
$$

We therefore can extract the density $\rho(z)$ and finally obtain the spectral representation:

$$
\frac{1}{(\sqrt{-z})^{\alpha}}=\int_{0}^{+\infty} d t \frac{1}{\pi} \sin \left(\frac{\pi \alpha}{2}\right)(\sqrt{t})^{-\alpha} \frac{1}{-z+t} .
$$

Going back to the $p^{2}$ variable, the fractional propagator can then be written as $t \rightarrow m^{2}$ :

$$
\frac{1}{\left(p^{2}\right)^{\alpha / 2}}=\int_{0}^{+\infty} d m \frac{2}{\pi} \sin \left(\frac{\pi \alpha}{2}\right) m^{1-\alpha} \frac{1}{p^{2}+m^{2}},
$$

and we obtain that the fractional propagator is a continuous distribution of scalar massive propagators with mass parameter $m$ weighed by a spectral density $\propto m^{1-\alpha}$. It is easily shown that in the limit $\alpha \rightarrow 2^{-}$(or $d_{\mathcal{U}} \rightarrow 1^{+}$) the right-hand side of Eq. (66) reduces to the massless scalar propagator $1 / p^{2}$ as upon putting $\alpha=2-\epsilon$ one obtains $\left[\frac{2}{\pi} \sin \left(\frac{\pi \alpha}{2}\right) m^{1-\alpha}\right]_{\alpha=2-\epsilon} \rightarrow \epsilon m^{-1+\epsilon} \rightarrow 2 \delta(m)$ on account of the Dirac $\delta$-function representation $\delta(x)=\lim _{\epsilon \rightarrow 0} \frac{\epsilon}{2}|x|^{-1+\epsilon}$ (the factor 2 is canceled by the fact that the integral in $d m$ ranges only over half the real axis).

We will show explicitly in the following that it is possible to obtain the same result for the propagator starting from the $(d+1)$ local theory.

Using the field decomposition in (46) we now compute the bulk Wightman function for the field $\Phi(x, y)$ 


$$
\begin{aligned}
\left\langle 0\left|\Phi(x, y) \Phi\left(x^{\prime}, y^{\prime}\right)\right| 0\right\rangle= & \frac{1}{2} \int \frac{d^{3} \boldsymbol{p}}{(2 \pi)^{3}} \int d \lambda \mu(\lambda) \int \frac{d^{3} \boldsymbol{p}^{\prime}}{(2 \pi)^{3}} \int d \lambda^{\prime} \mu\left(\lambda^{\prime}\right) \\
& \times\langle 0|\left[a(\boldsymbol{p}, \lambda) \frac{e^{i\left[\boldsymbol{p} \cdot \boldsymbol{x}-\omega_{\lambda^{2}}(\boldsymbol{p}) t\right]}}{\sqrt{\omega_{\lambda^{2}}(\boldsymbol{p})}} \psi(y, \lambda)+a^{\dagger}(\boldsymbol{p}, \lambda) \frac{e^{-i\left[\boldsymbol{p} \cdot \boldsymbol{x}-\omega_{\lambda^{2}}(\boldsymbol{p}) t\right]}}{\sqrt{\omega_{\lambda^{2}(\boldsymbol{p})}}} \psi^{*}(y, \lambda)\right] \\
& \times\left[a\left(\boldsymbol{p}^{\prime}, \lambda^{\prime}\right) \frac{e^{i\left[\boldsymbol{p}^{\prime} \cdot \boldsymbol{x}^{\prime}-\omega_{\lambda^{\prime 2}}\left(\boldsymbol{p}^{\prime}\right) t^{\prime}\right]}}{\sqrt{\omega_{\lambda^{\prime 2}\left(\boldsymbol{p}^{\prime}\right)}}} \psi\left(y^{\prime}, \lambda^{\prime}\right)+a^{\dagger}\left(\boldsymbol{p}^{\prime}, \lambda^{\prime}\right) \frac{e^{-i\left[\boldsymbol{p}^{\prime} \cdot \boldsymbol{x}^{\prime}-\omega_{\lambda^{\prime 2}}\left(\boldsymbol{p}^{\prime}\right) t^{\prime}\right]}}{\sqrt{\omega_{\lambda^{\prime 2}}\left(\boldsymbol{p}^{\prime}\right)}} \psi^{*}\left(y^{\prime}, \lambda^{\prime}\right)\right]|0\rangle .
\end{aligned}
$$

Expanding the product of the two factors in square parenthesis, when taking the vacuum expectation value, we get a nonzero contribution only from the cross term of the type $a(\boldsymbol{p}, \lambda) a^{\dagger}\left(\boldsymbol{p}^{\prime}, \lambda^{\prime}\right)$ :

$$
\begin{aligned}
\left\langle 0\left|\Phi(x, y) \Phi\left(x^{\prime}, y^{\prime}\right)\right| 0\right\rangle= & \frac{1}{2} \int \frac{d^{3} \boldsymbol{p}}{(2 \pi)^{3}} \int \frac{d^{3} \boldsymbol{p}^{\prime}}{(2 \pi)^{3}} \int d \lambda \mu(\lambda) \int d \lambda^{\prime} \mu\left(\lambda^{\prime}\right) \\
& \times\left\langle 0\left|a(\boldsymbol{p}, \lambda) a^{\dagger}\left(\boldsymbol{p}^{\prime}, \lambda^{\prime}\right)\right| 0\right\rangle \frac{e^{+i\left[\boldsymbol{p} \cdot \boldsymbol{x}-\omega_{\lambda^{2}}(\boldsymbol{p}) t\right]}}{\sqrt{\omega_{\lambda^{2}}(\boldsymbol{p})}} \frac{e^{-i\left[\boldsymbol{p}^{\prime} \cdot \boldsymbol{x}^{\prime}-\omega_{\lambda^{\prime 2}}\left(\boldsymbol{p}^{\prime}\right) t^{\prime}\right]}}{\sqrt{\omega_{\lambda^{2}}\left(\boldsymbol{p}^{\prime}\right)}} \psi^{*}(y, \lambda) \psi\left(y^{\prime}, \lambda^{\prime}\right)
\end{aligned}
$$

and the vacuum expectation value $\left\langle 0\left|a(\boldsymbol{p}, \lambda) a^{\dagger}\left(\boldsymbol{p}^{\prime}, \lambda^{\prime}\right)\right| 0\right\rangle$ can be computed from the identity:

$$
\left\langle 0\left|a(\boldsymbol{p}, \lambda) a^{\dagger}\left(\boldsymbol{p}^{\prime}, \lambda^{\prime}\right)\right| 0\right\rangle=\left\langle 0\left|\left[a(\boldsymbol{p}, \lambda), a^{\dagger}\left(\boldsymbol{p}^{\prime}, \lambda^{\prime}\right)\right]+a^{\dagger}\left(\boldsymbol{p}^{\prime}, \lambda^{\prime}\right) a(\boldsymbol{p}, \lambda)\right| 0\right\rangle=\left\langle 0\left|\left[a(\boldsymbol{p}, \lambda), a^{\dagger}\left(\boldsymbol{p}^{\prime}, \lambda^{\prime}\right)\right]\right| 0\right\rangle .
$$

Using the quantization rules given in Eqs. (47a)-(47c) we find

$$
\left\langle 0\left|a(\boldsymbol{p}, \lambda) a^{\dagger}\left(\boldsymbol{p}^{\prime}, \lambda^{\prime}\right)\right| 0\right\rangle=(2 \pi)^{3} \delta^{3}\left(\boldsymbol{p}-\boldsymbol{p}^{\prime}\right) \frac{1}{\mu(\lambda)} \delta\left(\lambda-\lambda^{\prime}\right)
$$

and, using the result in Eq. (70), we find

$$
\begin{aligned}
\left\langle 0\left|\Phi(x, y) \Phi\left(x^{\prime}, y^{\prime}\right)\right| 0\right\rangle= & \frac{1}{2} \int \frac{d^{3} \boldsymbol{p}}{(2 \pi)^{3}} \int \frac{d^{3} \boldsymbol{p}^{\prime}}{(2 \pi)^{3}} \int d \lambda \mu(\lambda) \int d \lambda^{\prime} \mu\left(\lambda^{\prime}\right) \frac{e^{+i\left[\boldsymbol{p} \cdot \boldsymbol{x}-\omega_{\lambda^{2}}(\boldsymbol{p}) t\right]}}{\sqrt{\omega_{\lambda^{2}}(\boldsymbol{p})}} \frac{e^{-i\left[\boldsymbol{p}^{\prime} \cdot \boldsymbol{x}^{\prime}-\omega_{\lambda^{\prime 2}}\left(\boldsymbol{p}^{\prime}\right) t^{\prime}\right]}}{\sqrt{\omega_{\lambda^{\prime 2}}\left(\boldsymbol{p}^{\prime}\right)}} \psi^{*}(y, \lambda) \psi\left(y^{\prime}, \lambda^{\prime}\right) \\
& \times(2 \pi)^{3} \delta^{3}\left(\boldsymbol{p}-\boldsymbol{p}^{\prime}\right) \frac{1}{\mu(\lambda)} \delta\left(\lambda-\lambda^{\prime}\right) .
\end{aligned}
$$

Then, performing the integrations in $\boldsymbol{p}^{\prime}$ and $\lambda^{\prime}$ by making use of the delta functions we obtain

$$
\left\langle 0\left|\Phi(x, y) \Phi\left(x^{\prime}, y^{\prime}\right)\right| 0\right\rangle=\int \frac{d^{3} \boldsymbol{p}}{(2 \pi)^{3}} \int_{0}^{+\infty} d \lambda \mu(\lambda) \frac{e^{i \boldsymbol{p} \cdot\left(\boldsymbol{x}-\boldsymbol{x}^{\prime}\right)} e^{-i \omega_{\lambda^{2}}(\boldsymbol{p})\left(t-t^{\prime}\right)}}{2 \omega_{\lambda^{2}}(\boldsymbol{p})} \psi^{*}(y, \lambda) \psi\left(y^{\prime}, \lambda\right) .
$$

The integral over $\boldsymbol{p}$ gives, on account of Eq. (56), the Wightman function [35] of a scalar field of mass $\lambda$,

$$
W_{\lambda^{2}}\left(x, x^{\prime}\right)=\int \frac{d^{3} \boldsymbol{p}}{(2 \pi)^{3}} \frac{e^{-i p \cdot\left(x-x^{\prime}\right)}}{2 \omega_{\lambda^{2}}(\boldsymbol{p})}=\int \frac{d^{4} p}{(2 \pi)^{4}} \Theta\left(p_{0}\right) 2 \pi \delta\left(p^{2}-\lambda^{2}\right) e^{-i p \cdot\left(x-x^{\prime}\right)}
$$

where $W_{\lambda^{2}}(p)=\Theta\left(p^{0}\right) 2 \pi \delta\left(p^{2}-\lambda^{2}\right)$ is the Fourier-space Wightman function of mass $\lambda$. The bulk two-point function can therefore be written as

$$
\left\langle 0\left|\Phi(x, y) \Phi\left(x^{\prime}, y^{\prime}\right)\right| 0\right\rangle=\int_{0}^{\infty} d \lambda \mu(\lambda) W_{\lambda^{2}}\left(x, x^{\prime}\right) \psi^{*}(y, \lambda) \psi\left(y^{\prime}, \lambda\right) .
$$

The brane induced Wightman function of the nonlocal field $\phi(x)$, on account of the boundary conditions imposed on the wave functions, $\left.\psi(y, \lambda)\right|_{y=0}=1$, is

$$
\left\langle 0\left|\phi(x) \phi\left(x^{\prime}\right)\right| 0\right\rangle=\lim _{y, y^{\prime} \downarrow 0}\left\langle 0\left|\Phi(x, y) \Phi\left(x^{\prime}, y^{\prime}\right)\right| 0\right\rangle=\int_{0}^{\infty} d \lambda \mu(\lambda) W_{\lambda^{2}}\left(x, x^{\prime}\right) .
$$

Therefore, we can see that the two-point Wightman function for the brane field can be expressed as an integral over the mass of a Wightman function of an ordinary massive scalar field. 
We now make contact between our approach and that of Refs. [33,34] which fixes the normalization of a scalar field $O_{\mathcal{U}}(x)$ of scaling dimension $d_{\mathcal{U}}$ by comparison with the phase space of $n$ massless particles. Indeed, the correlation function for the field $O_{\mathcal{U}}(x)$ can be written in terms of a spectral density $\rho\left(P^{2}\right)$ :

$\left\langle 0\left|O_{\mathcal{U}}(x) O_{\mathcal{U}}^{\dagger}(0)\right| 0\right\rangle=\int \frac{d^{4} P}{(2 \pi)^{4}} e^{-i P \cdot x}\left|\left\langle 0\left|O_{\mathcal{U}}(0)\right| p\right\rangle\right|^{2} \rho\left(P^{2}\right)$

and on dimensional grounds, given that $O_{\mathcal{U}}$ has scaling dimension $d_{\mathcal{U}}$, one can write

$$
\left|\left\langle 0\left|O_{\mathcal{U}}(0)\right| p\right\rangle\right|^{2} \rho\left(P^{2}\right)=A_{d_{\mathcal{U}}} \theta\left(P^{0}\right) \theta\left(P^{2}\right)\left(P^{2}\right)^{d_{\mathcal{U}}-2}
$$

with

$$
A_{d_{\mathcal{U}}}=\frac{16 \pi^{5 / 2}}{(2 \pi)^{2 d_{\mathcal{U}}}} \frac{\Gamma\left(d_{\mathcal{U}}+1 / 2\right)}{\Gamma\left(d_{\mathcal{U}}-1\right) \Gamma\left(2 d_{\mathcal{U}}\right)}
$$

a numerical constant that has been related in $[33,34]$ to the phase space factor of a fractional number $\left(d_{\mathcal{U}}\right)$ of massless particles.

The field $O_{\mathcal{U}}(x)$ must coincide, up to a numerical constant, with the field defined within our approach, à la Caffarelli-Silvestre, by $\phi(x)=\lim _{y \rightarrow 0^{+}} \Phi(x, y)$, with $\alpha=$ $4-2 d_{\mathcal{U}}$ so that we can put:

$$
\phi(x)=C^{\prime} O_{\mathcal{U}}(x) .
$$

By using Eq. (73) into Eq. (75) we can write the two-point Wightman function of the (neutral) field $\phi(x)$ as

$$
\langle 0|\phi(x) \phi(0)| 0\rangle=\int \frac{d^{4} P}{(2 \pi)^{4}} e^{-i P \cdot x} 2 \pi \theta\left(P^{0}\right) \theta\left(P^{2}\right) \frac{\mu\left(\sqrt{P^{2}}\right)}{2 \sqrt{P^{2}}} .
$$

Then, making use of the explicit expression of the function $\mu(\lambda)$ given in Eq. (44) and comparing with Eqs. (76) and (77) allows us to extract the relation between the constant $C^{\prime}$ and the quantity $A_{d_{\mathcal{U}}}$ :

$$
\left.\left[\frac{2^{-\alpha / 2} \pi}{\Gamma(\alpha / 2) \sin (\pi \alpha / 2)}\right]^{-2}\right|_{\alpha=4-2 d_{\mathcal{U}}}=\left(C^{\prime}\right)^{2} \frac{1}{\pi} A_{d_{\mathcal{U}}} .
$$

Numerically it is easily found that

$$
\left(C^{\prime}\right)^{2}=(2 \pi)^{2-\alpha}(2-\alpha)
$$

We note that the same reasoning leading to Eq. (75) would allow us to establish the same identity for the timeordered propagator.
Indeed, the time-ordered product of the local fields is

$$
\begin{aligned}
\langle 0| T[ & \left.\Phi(x, y) \Phi\left(x^{\prime}, y^{\prime}\right)\right]|0\rangle \\
= & \int_{0}^{\infty} d \lambda \mu(\lambda) \psi^{*}(y, \lambda) \psi\left(y^{\prime}, \lambda\right) \\
& \times \int \frac{d^{3} \boldsymbol{p}}{(2 \pi)^{3}} \frac{e^{-i p \cdot\left(x-x^{\prime}\right)}}{2 \omega_{\lambda^{2}}(\boldsymbol{p})}\left[e^{-i \omega_{\lambda^{2}}(\boldsymbol{p}) \tau} \theta\left(t-t^{\prime}\right)\right. \\
& \left.\left.+e^{+i \omega_{\lambda^{2}}(\boldsymbol{p}) \tau} \theta\left(t^{\prime}-t\right)\right)\right]
\end{aligned}
$$

and using the integral representation of the Heaviside $\theta$ function

$$
\begin{aligned}
\langle 0| T & {\left[\Phi(x, y) \Phi\left(x^{\prime}, y^{\prime}\right)\right]|0\rangle } \\
= & \int_{0}^{\infty} d \lambda \mu(\lambda) \psi^{*}(y, \lambda) \psi\left(y^{\prime}, \lambda\right) \\
& \times \lim _{\epsilon \rightarrow 0} \int \frac{d^{3} \boldsymbol{p}}{(2 \pi)^{3}} \frac{d \omega}{2 \pi} \frac{i e^{i\left[\omega\left(t-t^{\prime}\right)-p \cdot\left(x-x^{\prime}\right)\right]}}{\omega^{2}-\omega_{\lambda^{2}}^{2}+i \epsilon}
\end{aligned}
$$

or, using the momentum four-vector $k=(\omega, \boldsymbol{p})$

$$
\begin{aligned}
& \left\langle 0\left|T\left[\Phi(x, y) \Phi\left(x^{\prime}, y^{\prime}\right)\right]\right| 0\right\rangle \\
& \quad=\lim _{\epsilon \rightarrow 0} \int_{0}^{\infty} d \lambda \mu(\lambda) \psi^{*}(y, \lambda) \psi\left(y^{\prime}, \lambda\right) \int \frac{d^{4} k}{(2 \pi)^{4}} \frac{i e^{i k \cdot\left(x-x^{\prime}\right)}}{k^{2}-\lambda^{2}+i \epsilon} .
\end{aligned}
$$

Therefore, we get

$$
\begin{aligned}
&\langle 0| T {\left[\Phi(x, y) \Phi\left(x^{\prime}, y^{\prime}\right)\right]|0\rangle } \\
& \quad=\int_{0}^{\infty} d \lambda \mu(\lambda) \psi^{*}(y, \lambda) \psi\left(y^{\prime}, \lambda\right) D_{F}\left(x-x^{\prime}, \lambda^{2}\right)
\end{aligned}
$$

where we have introduced the Feynman propagator of a scalar field of mass $m$

$$
D_{F}\left(x-x^{\prime}, \lambda^{2}\right)=\lim _{\epsilon \rightarrow 0} \int \frac{d^{4} k}{(2 \pi)^{4}} \frac{i e^{i k \cdot\left(x-x^{\prime}\right)}}{k^{2}-\lambda^{2}+i \epsilon} .
$$

Now, from Eq. (86) we can extract the propagator for the nonlocal theory by taking the brane limit that defines the field $\phi(x)=\lim _{y \rightarrow 0} \Phi(x, y)$, therefore

$$
\left\langle 0\left|T\left[\phi(x) \phi\left(x^{\prime}\right)\right]\right| 0\right\rangle=\lim _{y, y^{\prime} \rightarrow 0}\left\langle 0\left|T\left[\Phi(x, y) \Phi\left(x^{\prime}, y^{\prime}\right)\right]\right| 0\right\rangle .
$$

Since we have that the wave functions in (86) satisfy the boundary conditions (30), we finally have

$$
\left\langle 0\left|T\left[\phi(x) \phi\left(x^{\prime}\right)\right]\right| 0\right\rangle=\int_{0}^{\infty} d \lambda \mu(\lambda) D_{F}\left(x-x^{\prime} ; \lambda^{2}\right) .
$$

Now, using (44) and taking the Fourier transform of Eq. (89), we can find the momentum space propagator $D(p)$ of the brane nonlocal field, $\phi(x)$, as 
$D(p)=\frac{i}{2}\left[\frac{2^{-\frac{\alpha}{2}} \pi}{\Gamma\left(\frac{\alpha}{2}\right) \sin \left(\pi \frac{\alpha}{2}\right)}\right]^{-2} \int_{0}^{\infty} d \lambda^{2}\left(\lambda^{2}\right)^{-\alpha / 2} \frac{1}{p^{2}-\lambda^{2}+i \epsilon}$.

We remark, first of all, that if the result of Eq. (90) is Wick rotated it coincides, up to an immaterial normalization constant, with the result obtained in Sec. III D [see Eq. (66)] for the fractional propagator, then the two propagators are characterized by the same particle content. Equation (90) is then to be compared with the propagator of the unparticle field of dimension $d_{\mathcal{U}}$ [34] given as [36]

$D_{U}(p)=i \frac{A_{d_{\mathcal{U}}}}{2 \pi} \int_{0}^{\infty} d M^{2}\left(M^{2}\right)^{d_{\mathcal{U}}-2} \frac{1}{p^{2}-M^{2}+i \epsilon}$.

By comparing Eq. (90) to Eq. (91) in order for the two propagators to satisfy $D(p)=\left(C^{\prime}\right)^{2} D_{U}(p)$, which follows from Eq. (79), we must have, for the constant $\left(C^{\prime}\right)^{2}$, again Eq. (81). The constant $\left(C^{\prime}\right)^{2}$ can be computed on account of the relation $\alpha=4-2 d_{\mathcal{U}}$ and we find again, as expected, the same result of Eq. (82). Computing explicitly the integrations in Eq. (90) and Eq. (91) we find

$$
\begin{aligned}
D(p) & =-i \frac{2^{\alpha-1} \sin \left(\frac{\pi \alpha}{2}\right) \Gamma\left(\frac{\alpha}{2}\right)^{2}}{\pi^{2}}\left(-p^{2}-i \epsilon\right)^{-\frac{\alpha}{2}} \\
D_{\mathcal{U}}(p) & =+i \frac{A_{d_{\mathcal{U}}}}{2 \sin \left(\pi d_{\mathcal{U}}\right)}\left(-p^{2}-i \epsilon\right)^{d_{\mathcal{U}}-2}
\end{aligned}
$$

which indeed differ exactly by the constant $\left(C^{\prime}\right)^{2}$ as in Eq. (82). Note that while Eq. (92b) in the limit $d_{\mathcal{U}} \rightarrow 1$ reproduces exactly the massless scalar field propagator $D_{\mathcal{U}}(p) \rightarrow+i /\left(p^{2}+i \epsilon\right)$, Eq. (92a) when $\alpha \rightarrow 2$ reproduces the same massless scalar propagator only up to the numerical constant $\left(C^{\prime}\right)^{2}: D(p) \rightarrow+i(2-\alpha) /\left(p^{2}+i \epsilon\right)$. It is thus clear the advantage of working with the normalization introduced by Georgi $[33,34]$ for the nonlocal unparticle field.

We conclude this section by showing explicitly how the extension problem straightforwardly provides the standard unparticle effective action routinely used in the phenomenology of this model. We start then from the central result of the extension problem, namely Eq. (21), which relates the local action in $(4+1)$ dimensions to a nonlocal action in four dimensions:

$$
S=\frac{1}{2 C} \int d^{4} x \phi(x) \square^{\alpha / 2} \phi(x) .
$$

Here we redefine the field using Eq. (79) and use the field $O_{\mathcal{U}}(x)$ normalized "à la Georgi," i.e., to have the same propagator computed in [34]. We then obtain

$$
S=\frac{\left(C^{\prime}\right)^{2}}{C} \frac{1}{2} \int d^{4} x O_{\mathcal{U}}(x) \square^{\alpha / 2} O_{\mathcal{U}}(x)
$$

Then by using the explicit results derived above, cf. Eq. (81), together with Eq. (5) and upon the replacement $\alpha=4-2 d_{\mathcal{U}}$ we find

$$
S=\frac{2 \sin \left(\pi d_{\mathcal{U}}\right)}{A_{d_{\mathcal{U}}}} \frac{1}{2} \int d^{4} x O_{\mathcal{U}}(x) \square^{2-d_{\mathcal{U}}} O_{\mathcal{U}}(x) .
$$

This classical action being quadratic in the field is equivalent to the effective action and thus Eq. (95) is the effective action for unparticles. Indeed, its second order functional derivative with respect to the fields gives the inverse twopoint function according to

$\frac{\delta S}{\delta O_{\mathcal{U}}(x) \delta O_{\mathcal{U}}\left(x^{\prime}\right)}=i D_{\mathcal{U}}^{-1}\left(x, x^{\prime}\right)=\frac{2 \sin \left(\pi d_{\mathcal{U}}\right)}{A_{d_{\mathcal{U}}}} \square^{2-d_{\mathcal{U}}}$

which is consistent with the explicit computation of the propagator offered above, cf. Eq. (92b).

\section{VACUUM ENERGY DENSITY}

Here we discuss the brane vacuum energy of the nonlocal theory as obtained from the local Hamiltonian of the extended theory in $(d+1)$ dimensions. The bulk Hamiltonian operator $\mathcal{H}$ can be obtained as the component $T_{00}$ of the energy-momentum tensor $T_{\mu \nu}$ of the $(d+1)$ local theory:

$$
\begin{aligned}
\mathcal{H}= & \frac{1}{2} \int d^{3} \boldsymbol{x} \int_{0}^{+\infty} d y y^{1-\alpha}\left[\partial_{0} \Phi(x, y) \partial_{0} \Phi(x, y)\right. \\
& \left.-\Phi(x, y) \partial_{0}^{2} \Phi(x, y)\right]
\end{aligned}
$$

with $x=(\boldsymbol{x}, t)$. We start again from the field expansion in Eq. (46) and calculate the bulk energy as $E_{\text {vac }}^{\text {bulk }}=\langle 0|\mathcal{H}| 0\rangle$ that gives

$$
\begin{aligned}
E_{\mathrm{vac}}^{\text {bulk }}= & \frac{1}{2} \int d^{3} \boldsymbol{x} \int \frac{d^{3} \boldsymbol{p}}{(2 \pi)^{3}} \int_{0}^{+\infty} d \lambda \mu(\lambda) \omega_{\lambda^{2}}(\boldsymbol{p}) \\
& \times \int_{0}^{+\infty} d y y^{1-\alpha} \psi^{*}(y, \lambda) \psi(y, \lambda) .
\end{aligned}
$$

It can easily be verified, by taking into account the dimensions of the function $\mu(\lambda)$, that the above quantity is correctly dimensioned as an energy. From this bulk energy we ought to extract the vacuum brane energy $E_{\text {vac }}^{\text {brane }}$. One possibility is to think to slice up the extra space dimension $y \in[0,+\infty]$ and assign the first slice $y \in$ $\left[0, y_{\text {cut }}\right]$ to the brane. Note that in Eq. (98) the integration over the three-space entails an infinite factor. To avoid such an infinite factor as it is standard in quantum field theory, we imagine enclosing our brane on a large but finite 
volume $V$. This allows us to define a brane vacuum energy density:

$$
\begin{aligned}
\varepsilon_{\mathrm{vac}}= & \frac{1}{2} \int \frac{d^{3} \boldsymbol{p}}{(2 \pi)^{3}} \int_{0}^{+\infty} d \lambda \mu(\lambda) \omega_{\lambda^{2}}(\boldsymbol{p}) \\
& \times \int_{0}^{y_{\mathrm{cut}}} d y y^{1-\alpha} \psi^{*}(y, \lambda) \psi(y, \lambda) .
\end{aligned}
$$

The cutoff length $y_{\text {cut }}$ corresponds (in natural units) to an energy scale

$$
2 \pi \Lambda_{\mathcal{U}}=1 / y_{\text {cut }} .
$$

This physical cutoff defines, on the brane, an effective field theory which corresponds to integrating out, in Eq. (98), all the degrees of freedom (d.o.f.) at energies $E$ higher than $2 \pi \Lambda_{\mathcal{U}}\left(E \geq 2 \pi \Lambda_{\mathcal{U}}\right)$. Indeed the $y$ integral in Eq. (99) can be computed approximating the wave functions by means of the imposed boundary conditions $\psi(y, \lambda) \rightarrow 1$ as $y \rightarrow 0$ and converting it into an energy integral (over the variable $E=1 / y)$ :

$\varepsilon_{\mathrm{vac}}=\int_{0}^{+\infty} d \lambda \mu(\lambda) \int \frac{d^{3} \boldsymbol{p}}{(2 \pi)^{3}} \frac{\omega_{\lambda^{2}}(\boldsymbol{p})}{2} \int_{2 \pi \Lambda_{\mathcal{U}}}^{\infty} d E E^{\alpha-3}$

which shows clearly how the d.o.f. $E \geq 2 \pi \Lambda_{\mathcal{U}}$ are being integrated out. This defines an effective field theory valid up to energies $\approx 2 \pi \Lambda_{\mathcal{U}}$. The vacuum energy density on the brane is finally given by

$$
\varepsilon_{\mathrm{vac}}=\frac{1}{(2-\alpha)\left(2 \pi \Lambda_{\mathcal{U}}\right)^{2-\alpha}} \int_{0}^{+\infty} d \lambda \mu(\lambda) \int \frac{d^{3} \boldsymbol{p}}{(2 \pi)^{3}} \frac{\omega_{\lambda^{2}}(\boldsymbol{p})}{2} .
$$

Note that if $d_{\mathcal{U}}$ is the scaling dimension of the field then $\alpha=4-2 d_{\mathcal{U}}$ and $2-\alpha=2 d_{\mathcal{U}}-2>0$ for $d_{\mathcal{U}}>1$. We note that in Eq. (102) the fractional power of $\Lambda_{\mathcal{U}}$ exactly compensates the fractional dimensions of the function $\mu(\lambda) \propto \lambda^{1-\alpha}$ thus leaving a genuine energy density. As a final remark we note that Eq. (102) expresses the vacuum energy density of the nonlocal effective theory as an integral over the mass parameter $\lambda$ of the vacuum energy density of a scalar field of mass $\lambda$, since $\omega_{\lambda^{2}}(\boldsymbol{p}) / 2$ is the zero point energy of a mode of momentum $\boldsymbol{p}$ and mass $\lambda$, weighted by the function $\mu(\lambda)$. Equation (102) coincides up to a numerical constant with the central result of Ref. [37] for the vacuum energy of unparticles: it is still a divergent quantity but it can, for instance, be used to compute the unparticle Casimir energy by imposing appropriate geometrical boundary conditions [37]. Note that Eq. (102) can be cast in the standard normalization routinely used for unparticles (cf. $[33,34]$ ) by using the result in Eq. (81). Namely,
$\mu(\lambda)=\left(C^{\prime}\right)^{2} \frac{A_{d_{\mathcal{U}}}}{\pi} \lambda^{1-\alpha}=(2 \pi)^{2-\alpha}(2-\alpha) \frac{A_{d_{\mathcal{U}}}}{\pi} \lambda^{1-\alpha}$

and then by making use of Eq. (82) we find $\left(2-\alpha=2 d_{\mathcal{U}}-2\right)$

$$
\varepsilon_{\mathrm{vac}}=\frac{A_{d_{\mathcal{U}}}}{\pi \Lambda_{\mathcal{U}}^{2 d_{\mathcal{U}}-2}} \int_{0}^{+\infty} d \lambda \lambda^{2 d_{\mathcal{U}}-3} \int \frac{d^{3} \boldsymbol{p}}{(2 \pi)^{3}} \frac{\omega_{\lambda^{2}}(\boldsymbol{p})}{2},
$$

and this coincides with the central result derived in [37] for the UnCasimir effect.

\section{NEGATIVE POWERS OF THE FRACTIONAL LAPLACIAN}

In [6], it has been shown that the extension problem in the original formulation of Eq. (4) can be directly applied to the inverse powers of a generic second order differential operator (i.e., $\Delta^{-\alpha / 2}$ with $\alpha>0$ ). Interestingly, it has also been discussed in [38] that the same extension problem is valid for negative powers of the fractional Laplacian but mapping the Dirichlet boundary condition to a Neumann boundary condition.

In particular, for $\alpha \in(0,2)$ and $f: \mathbb{R}^{n} \rightarrow \mathbb{R}$, a smooth and bounded function, one can consider the extension problem in the upper half-space with boundary conditions of the Neumann-type as follows:

$$
\begin{gathered}
\frac{\partial w}{\partial y^{\alpha}}(x, 0)=-f(x) \quad x \in \mathbb{R}^{n}, \\
\nabla \cdot\left(y^{1-\alpha} \nabla w\right)=0 \quad x \in \mathbb{R}^{n}, y>0 .
\end{gathered}
$$

Note that in order to have a well-posed problem, an extra condition should be imposed to avoid the fact that if $w$ is a solution, then $w+c$ is also a solution for any $c \in \mathbb{R}$. Therefore, we need to assume the following boundary condition for large $y$ :

$$
\lim _{y \rightarrow \infty} w(x, y) \rightarrow 0
$$

Then the solution $w(x, y)$ of problems (105a) and (105b) that satisfies the boundary condition (106) has an explicit expression given by

$$
S_{n, \alpha}(-\Delta)^{-\alpha / 2} f(x)=\lim _{y \rightarrow 0^{+}} w(x, y)
$$

where $S_{n, \alpha}$ is a constant defined as

$$
S_{n, \alpha}=\frac{C_{n, 2-\alpha}}{(n-\alpha) D_{n, \alpha}}
$$

with 


$$
C_{n, 2-\alpha}:=\frac{2^{\alpha} \Gamma\left(\frac{n+\alpha}{2}\right)}{\pi^{n / 2} \Gamma\left(-\frac{\alpha}{2}\right)}, \quad D_{n, \sigma}:=\frac{\pi^{\alpha-n / 2}}{\Gamma\left(\frac{s}{2}\right)} \Gamma\left(\frac{n-s}{2}\right) .
$$

Thus, while the fractional Laplacian we used in the previous sections can be considered as an operator connecting the Dirichlet boundary conditions for the original problem to Neumann-type conditions for the "local" extended problem, (3a) and (3b); the inverse fractional Laplacian of this section is an operator that realizes the opposite mapping: from Neumann-type boundary conditions for the original problem to Dirichlet conditions, (105a) and (105b), in the local extended problem, at least for $\alpha \in(0,2)$.

We show here how this can be explicitly done in terms of the eigenfunctions of the problem given in Eq. (29). The boundary conditions on the eigenfunctions $\psi(\lambda, y)$ now become

$$
\psi(\lambda, 0)=0,\left.\quad y^{1-\alpha} \partial_{y} \psi(\lambda, y)\right|_{y=0}=1
$$

which from Eq. (33) can easily be shown to be solved by

$$
A=\frac{2^{\frac{\alpha}{2}} \Gamma\left(\frac{\alpha}{2}+1\right)}{\alpha \lambda^{\alpha}}, \quad B=0,
$$

which lead, through Eq. (29), to the solutions:

$$
\psi(\lambda, y)=\frac{2^{\frac{\alpha}{2}} \Gamma\left(\frac{\alpha}{2}+1\right)}{\alpha \lambda^{\alpha}}(\lambda y)^{\frac{\alpha}{2}} J_{\alpha / 2}(\lambda y) .
$$

It is important to note that we can apply here the same procedure as before because the local bulk field theory satisfies the same equation of motion (105b).

\section{DISCUSSION AND APPLICATIONS TO GENERAL RELATIVITY}

In the previous sections we have discussed particular aspects of a scalar nonlocal quantum field theory. Some properties of the theory can be obtained thinking about the nonlocal fields as living on a brane and studying a local bulk theory with an extra dimension. The features previously discussed can be applied to some extent to other fields and, in particular, a possible natural application would be to the so-called Dvali-Gabadadze-Porrati (DGP) model [39]. The DGP model is a possible way of describing the realization of a continuum of massive gravitons using extra dimensions and a brane on which the Standard Model is confined.

In particular the DGP model describes a $(3+1)$-dimensional brane living in a $(4+1)$-dimensional bulk spacetime, and the total action has both 5D and 4D parts, which reads schematically

$$
\begin{aligned}
S= & \frac{M_{5}^{3}}{2} \int d^{5} X \sqrt{-G} R(G)+\frac{M_{4}^{2}}{2} \int d^{4} x \sqrt{-g} R(g) \\
& +\int d^{4} x \mathcal{L}_{M}(g, \psi)
\end{aligned}
$$

where the $5 \mathrm{D}$ bulk coordinates and the $5 \mathrm{D}$ metric are, respectively, $X^{A}$ and $G_{A B}(X)$ with $A, B=0,1 \ldots, 4$; the $4 \mathrm{D}$ brane coordinates and the $4 \mathrm{D}$ metric are, respectively, $x^{\mu}$ and $g_{\mu \nu}(x)$ with $\mu, \nu=0,1 \ldots, 3$; and the $\psi(x)$ are the $4 \mathrm{D}$ matter fields which are described by the Lagrangian $\mathcal{L}_{M}$ and are assumed to be confined to the $4 \mathrm{D}$ brane. The constants $M_{5}$ and $M_{4}$ are, respectively, the 5D and the 4D Plank masses.

Taking the expansion around flat space $g_{\mu \nu}=\eta_{\mu \nu}+h_{\mu \nu}$ (with $\operatorname{det} h_{\mu \nu}=h$ ) and fixing the proper gauge, one can write the effective 4D DGP action in the following way [40]:

$$
\begin{aligned}
S= & \int d^{4} x \frac{M_{4}^{2}}{4}\left[\frac{1}{2} h_{\mu \nu} \mathcal{E}^{\mu \nu, \alpha \beta} h_{\alpha \beta}-\frac{1}{2} m\left(h_{\mu \nu} \Delta h^{\mu \nu}-h \triangle h\right)\right] \\
& +\frac{1}{2} \int d^{4} x h_{\mu \nu} T^{\mu \nu}
\end{aligned}
$$

where $\mathcal{E}^{\mu \nu, \alpha \beta}$ is the massless graviton kinetic operator defined by

$$
S_{\text {massless graviton }}=\int d^{D} x \frac{1}{2} h_{\mu \nu} \mathcal{E}^{\mu \nu, \alpha \beta} h_{\alpha \beta}
$$

and

$\mathcal{E}_{\alpha \beta}^{\mu \nu}=\left(\eta_{\alpha}^{(\mu} \eta_{\beta}^{\nu)}-\eta^{\mu \nu} \eta_{\alpha \beta}\right) \square-2 \partial^{(\mu} \partial_{(\alpha} \eta_{\beta)}^{\nu)}+\partial^{\mu} \partial^{\nu} \eta_{\alpha \beta}+\partial_{\alpha} \partial_{\beta} \eta^{\mu \nu}$

and

$$
m \equiv \frac{2 M_{5}^{3}}{M_{4}^{2}}
$$

is the so-called DGP scale. Interestingly, the operator $\triangle$ is the (formal) square root of the four-dimensional Laplacian, i.e.,

$$
\triangle \equiv \sqrt{-\square}
$$

Therefore, the action (114) is of the Fierz-Pauli form with an operator dependent mass $m \triangle$. The operator (118) corresponds, in terms of our previous analysis, to the particular case $\alpha=1$ in (6) and is known as a resonance mass, or soft mass [40]. The particle content of the theory emerges clearly from a study of the propagator. By analyticity considerations (similar to those given in Sec. III D) the momentum part of the propagator can be 
reconstructed in terms of its poles and cuts and it is possible to write the following spectral representation:

$$
\begin{array}{r}
\frac{-i}{p^{2}+m \sqrt{p^{2}}}=\int_{0}^{\infty} d s \frac{-i}{p^{2}+s} \rho(s) \\
\rho(s)=\frac{m}{\pi \sqrt{s}\left(s+m^{2}\right)}>0
\end{array}
$$

which shows therefore how this theory contains a continuum of ordinary (nonghost, nontachyon) gravitons, with masses $(\sqrt{s})$ ranging from 0 to $\infty$. This is in line with what we would expect from the previous analysis using the extension problem for a nonlocal theory. The different form of the spectral function in Eq. (119b) relative to what we found in Eq. (90) is due to the fact that, as opposed to our nonlocal theory, Eq. (93), or equivalently the unparticle model, Eq. (95), the DGP model is not scale invariant: it is indeed defined by the scale $m$ obtained as in Eq. (117). The behavior in terms of a continuous spectrum of mass states is also understood as the result of the dimensional reduction of the noncompact fifth dimension, when the Kaluza-Klein tower collapses into a Kaluza-Klein continuum. We conclude this paragraph with a remark about two limiting cases of the representation in Eq. (119a). First, consider the $m \rightarrow 0$ limit: the left-hand side of Eq. (119a) evidently reduces to the massless propagator $-i / p^{2}$ and the same is obtained by noting that in the same limit $\rho(s) \rightarrow 2 \delta(s)$ and the factor of 2 is accounted for by the integration over half of the real axis. More interesting is perhaps the $m \rightarrow \infty$ limit (or $m^{2} \gg p^{2}$ ) which is obtained by exchanging the limit with the integration in the right-hand side of Eq. (119a):

$$
\frac{-i}{m \sqrt{p^{2}}}=\frac{1}{m} \int_{0}^{\infty} d s \frac{1}{\pi \sqrt{s}} \frac{-i}{p^{2}+s} .
$$

Canceling out the mass scale $m$ in the above Eq. (120) one is left precisely with Eq. (66) upon replacing $m$ into $\sqrt{s}$ reproducing therefore the fractional propagator with $\alpha=1$. Thus the $m \rightarrow \infty$ limit in Eq. (119a) amounts to going from a theory explicitly dependent on a scale $m$ over to a scale invariant theory ( $m$ independent). The specific spectral density $1 /(\pi \sqrt{s})$ is thus clearly connected to the scale invariance of the fractional propagator $-i / \sqrt{p^{2}}$. This in retrospect means therefore that, for generic $\alpha$, the specific form of the function $\mu(\lambda) \propto \lambda^{1-\alpha}$ given in Eq. (44) in order to obtain the completeness relation, Eq. (45), is ultimately closely related to the request of scale invariance. Note that the representation in Eq. (120) coincides identically (up to a normalization constant) with Eq. (90) for $\alpha=1$ and $\lambda^{2} \rightarrow s$ and a Wick rotation.

Another interesting connection with general relativity and cosmology can be obtained noting that the Proca Lagrangian that describes the three d.o.f. of a massive photon

$$
\mathcal{L}=-\frac{1}{4} F_{\mu \nu} F^{\mu \nu}-\frac{1}{2} m_{\gamma}^{2} A_{\mu} A^{\mu}
$$

has been shown to be equivalent to a gauge-invariant but nonlocal Lagrangian given by (see [18])

$$
\mathcal{L}=-\frac{1}{4} F_{\mu \nu}\left(1-\frac{m_{\gamma}^{2}}{\square}\right) F^{\mu \nu}
$$

and therefore can be an interesting test bed for the strategy proposed in the previous section: rewrite a nonlocal action as a local action in $d+1$ dimensions using the extension problem, perform the calculations in this local setting and then read the result for the nonlocal action on the boundary. Moreover, it is a way to understand a more difficult nonlocal invariant like $(\square)^{-n}$. In the case of the Proca action we know that the final result should be a massive propagator. The presence of nonlocal terms like inverse powers of the d'Alambertian can be interesting because these operators become relevant in the IR and therefore can have consequences in cosmology. Recent models are discussed [16,41-43]. In particular, the model proposed in [16] that considers nonlocal infrared corrections via the inverse Laplacian is promising for the cosmological implications while passing all the important tests at solar system scales [41].

\section{CONCLUSIONS}

In this paper, we have analyzed in detail the quantum aspects of a nonlocal fractional theory on the brane from the point of view of a (local) scalar bulk quantum field theory with a boundary using the well-known and well-defined mathematical framework of the "extension problem" developed by Caffarelli and Silvestre. In particular, we have focused on a nonlocal theory on the brane [for example a $(3+1)$-dimensional spacetime] defined in terms of the fractional Laplacian and studied it from the point of view of the local theory in the bulk- $(4+1)$-dimensional-with one additional transverse spacelike dimension $(y)$. The problem is how to characterize the bulk fields that can describe the behavior of the nonlocal theory on the boundary. This is achieved by solving the equation of motion in the bulk separating out the transverse dimension (y), solving explicitly for the related eigenfunctions and imposing appropriate boundary conditions. We have then discussed the quantization of the local action in $(4+1)$ dimensions via the operator formalism. The bulk field $\Phi(x, y)$ in $(4+1)$ dimensions is first decomposed into normal modes separating out the ordinary spacetime coordinates $(x)$ from the extra spacelike coordinate $y$. Explicit expressions for the wave functions in the extra coordinate $y$ are given, which satisfy the appropriate boundary conditions whose completeness relation allows us to show that the bulk field obeys canonical equal-time commutations relations. On the other end, the study of the 
causal structure shows that while local commutativitythe vanishing of the commutator of fields for spacelike separation-is lost on the bulk it is restored on the brane. Indeed, the nonlocal field $\phi(x)$ obtained by the brane limit, $y \rightarrow 0$, satisfies local commutativity. We finally computed the two-point bulk correlation functions that induce, on the brane $(y \rightarrow 0$ limit), the expected nonlocal correlations functions. The nonlocal propagators thus found are shown to be equivalent to those obtained by other methods, namely, the computation of the spectral density representation in terms of discontinuities across the branch cut of the fractional propagator.

We find that what emerges naturally from the standard quantization of the bulk theory via the canonical operator formalism is that the two-point Wightman function (or Feynman propagator) for the brane field can be expressed as an integral over the mass of the corresponding Wightman function (or Feynman propagator) of an ordinary massive scalar field with a specific spectral density. This allows us to make a connection with the scale invariant theory proposed by Georgi (unparticle model), showing that the two approaches are characterized by the same particle content and are therefore equivalent.

We have also discussed the brane vacuum energy of the nonlocal fractional theory as obtained from the local Hamiltonian of the extended theory in $(d+1)$ dimensions. The bulk Hamiltonian operator $\mathcal{H}$ can be obtained as the component $T_{00}$ of the energy-momentum tensor $T_{\mu \nu}$ of the $(d+1)$ local theory. Then, one can extract the vacuum brane energy, $E_{\text {vac }}^{\text {brane }}$, from its bulk counterpart. This has been done, using an effective field theory approach, therefore integrating out the d.o.f. above a certain energy scale $\left(\Lambda_{\mathcal{U}}\right)$ defined as the inverse of the extra-dimension cutoff $y_{\text {cut }}$. This physical cutoff defines, on the brane, an effective field theory up to the energy $\Lambda_{\mathcal{U}}$.

We also extended the derivation of the eigenfunctions to the case of negative powers of the fractional Laplacian. The solution can be explicitly found in terms of the eigenfunctions of the initial problem changing the boundary conditions.

It is the authors' opinion that approaching the quantization of nonlocal fractional field theories via the extension problem should be further explored (for instance with respect to vector and/or spinor fields) and exploited to study possible applications in general relativity and cosmology.

\section{ACKNOWLEDGMENTS}

The work of A.M.F. was supported by a Swiss Government Excellence Scholarship and is currently supported from ERC Advanced Grant No. GravBHs-692951 and Ministerio de Educación y Ciencia Grant No. FPA201676005-C2-2-P. The authors wish to thank Michele Maggiore for many detailed and useful discussions.
[1] C. Bjorland, L. Caffarelli, and A. Figalli, Non-local gradient dependent operators, Adv. Math. 230, 1859 (2012).

[2] B. Barrios, A. Figalli, and X. Ros-Oton, Global regularity for the free boundary in the obstacle problem for the fractional Laplacian, Am. J. Math. 140, 415 (2018).

[3] L. Silvestre, Regularity of the obstacle problem for a fractional power of the laplace operator, Commun. Pure Appl. Math. 60, 67 (2007).

[4] C. Bjorland, L. Caffarelli, and A. Figalli, Nonlocal tug-ofwar and the infinity fractional Laplacian, Commun. Pure Appl. Math. 65, 337 (2012).

[5] M. Kwaśnicki, Ten equivalent definitions of the fractional laplace operator, Fractional Calculus Appl. Anal. 20, 7 (2017).

[6] P. R. Stinga and J. L. Torrea, Extension problem and harnack's inequality for some fractional operators, Commun. Partial Differ. Equations 35, 2092 (2010).

[7] L. Caffarelli and L. Silvestre, An extension problem related to the fractional Laplacian, Commun. Partial Differ. Equations 32, 1245 (2007).

[8] S.-Y. A. Chang and M. del Mar González, Fractional Laplacian in conformal geometry, Adv. Math. 226, 1410 (2011).

[9] A. Sapora, P. Cornetti, and A. Carpinteri, Wave propagation in nonlocal elastic continua modelled by a fractional calculus approach, Commun. Nonlinear Sci. Numer. Simul. 18, 63 (2013).

[10] M. Di Paola and M. Zingales, Long-range cohesive interactions of non-local continuum faced by fractional calculus, Int. J. Solids Struct. 45, 5642 (2008).

[11] S. Longhi, Fractional Schrödinger equation in optics, Opt. Lett. 40, 1117 (2015).

[12] G. La Nave and P. W. Phillips, Geodesically complete metrics and boundary non-locality in holography: Consequences for the entanglement entropy, Phys. Rev. D 94, 126018 (2016).

[13] K. Limtragool and P. W. Phillips, Anomalous dimension of the electrical current in strange metals from the fractional Aharonov-Bohm effect, Europhys. Lett. 121, 27003 (2018).

[14] G. La Nave and P. Phillips, Anomalous dimensions for boundary conserved currents in holography via the Caffarelli-Silvestre mechanism for p-forms, Commun. Math. Phys. 366, 119 (2019).

[15] G. La Nave, K. Limtragool, and P. W. Phillips, Colloquium: Fractional electromagnetism in quantum matter and highenergy physics, Rev. Mod. Phys. 91, 021003 (2019).

[16] M. Maggiore, Phantom dark energy from nonlocal infrared modifications of general relativity, Phys. Rev. D 89, 043008 (2014). 
[17] M. Maggiore and M. Mancarella, Nonlocal gravity and dark energy, Phys. Rev. D 90, 023005 (2014).

[18] E. Belgacem, Y. Dirian, S. Foffa, and M. Maggiore, Nonlocal gravity. Conceptual aspects and cosmological predictions, J. Cosmol. Astropart. Phys. 03 (2018) 002.

[19] N. Arkani-Hamed, S. Dimopoulos, G. Dvali, and G. Gabadadze, Non-local modification of gravity and the cosmological constant problem, arXiv:hep-th/0209227.

[20] A. O. Barvinsky, Nonlocal action for long-distance modifications of gravity theory, Phys. Lett. B 572, 109 (2003).

[21] S. Deser and R. P. Woodard, Nonlocal Cosmology, Phys. Rev. Lett. 99, 111301 (2007).

[22] J. Gomis, K. Kamimura, and J. Llosa, Hamiltonian formalism for space-time noncommutative theories, Phys. Rev. D 63, 045003 (2001).

[23] G. Calcagni, M. Montobbio, and G. Nardelli, Route to nonlocal cosmology, Phys. Rev. D 76, 126001 (2007).

[24] G. Calcagni, M. Montobbio, and G. Nardelli, Localization of nonlocal theories, Phys. Lett. B 662, 285 (2008).

[25] G. Calcagni and G. Nardelli, Nonlocal gravity and the diffusion equation, Phys. Rev. D 82, 123518 (2010).

[26] G. Calcagni, Taming the beast: Diffusion method in nonlocal gravity, Universe 4, 95 (2018); Correction: Calcagni, G. Taming the beast: Diffusion method in nonlocal gravity, Universe 5, 121(E) (2019).

[27] M. A. Rajabpour, Conformal symmetry in non-local field theories, J. High Energy Phys. 06 (2011) 076.

[28] M. Mintchev, The role of initial conditions in presence of extra dimensions, Phys. Lett. B 524, 363 (2002).

[29] M. Mintchev, Local fields on the brane induced by nonlocal fields in the bulk, Classical Quantum Gravity 18, 4801 (2001).
[30] M. Presilla, O. Panella, and P. Roy, Quantum phase transitions of the Dirac oscillator in the anti-Snyder model, Phys. Rev. D 92, 045019 (2015).

[31] F. W. Olver, D. W. Lozier, R. F. Boisvert, and C. W. Clark, NIST Handbook of Mathematical Functions, 1st ed (Cambridge University Press, New York, 2010).

[32] In relation to the notation in Sec. III D, this means that the identity holds for $d_{\mathcal{U}}>1$.

[33] H. Georgi, Unparticle Physics, Phys. Rev. Lett. 98, 221601 (2007).

[34] H. Georgi, Another odd thing about unparticle physics, Phys. Lett. B 650, 275 (2007).

[35] M. E. Peskin and D. V. Schroeder, An Introduction to Quantum Field Theory (Addison-Wesley, Reading, MA, 1995).

[36] See eq. 3 in [34].

[37] A. M. Frassino, P. Nicolini, and O. Panella, Unparticle Casimir effect, Phys. Lett. B 772, 675 (2017).

[38] F. del Teso, Theoretical, and numerical aspects for nonlocal equations of porous medium type, Ph.D. thesis, Universidad Autónoma de Madrid, https://repositorio.uam.es/handle/ 10486/669713.

[39] G. R. Dvali, G. Gabadadze, and M. Porrati, 4D gravity on a brane in 5D Minkowski space, Phys. Lett. B 485, 208 (2000).

[40] K. Hinterbichler, Theoretical aspects of massive gravity, Rev. Mod. Phys. 84, 671 (2012).

[41] E. Belgacem, A. Finke, A. Frassino, and M. Maggiore, Testing nonlocal gravity with Lunar Laser Ranging, J. Cosmol. Astropart. Phys. 02 (2019) 035.

[42] S. Deser and R. P. Woodard, Nonlocal cosmology II. Cosmic acceleration without fine tuning or dark energy, J. Cosmol. Astropart. Phys. 06 (2019) 034.

[43] L. Amendola, Y. Dirian, H. Nersisyan, and S. Park, Observational constraints in nonlocal gravity: the DeserWoodard case, J. Cosmol. Astropart. Phys. 03 (2019) 045. 